\title{
Letter:
}

\section{Effects of EDTA irrigation and water storage on the bonding durability of different adhesive resin cements to intra-radicular dentin*}

\author{
Cai-yun MAO ${ }^{1}$, Jia-jia $\mathrm{ZHAO}^{2}$, Wei $\mathrm{WANG}^{1}$, \\ Xin-hua GU $\mathrm{GH}^{\dagger+1}$ \\ ( ${ }^{I}$ Department of Stomatology, the First Affiliated Hospital, School of \\ Medicine, Zhejiang University, Hangzhou 310003, China) \\ $\left({ }^{2}\right.$ Department of Stomatology, Zhuji People's Hospital of Zhejiang \\ Province, Zhuji 311800, China) \\ ${ }^{\dagger}$ E-mail: guxh@zju.edu.cn \\ Received Aug. 24, 2013; Revision accepted Feb. 23, 2014 \\ Crosschecked Mar. 24, 2014
}

doi: 10.1631 jzus.B1300227

Document code: A

CLC number: R783.1

One hundred and twenty single-root teeth were selected to evaluate the bonding durability of three different adhesive resin cements to intra-radicular dentin and the effect of ethylenediaminetetraacetic acid (EDTA) irrigation on bonding durability, over six months of water storage. We found that water storage, type of cement, post space pretreatment, and root region were significant factors affecting the push-out bond strength. Water storage reduced the bond strength of RelyX Unicem (RU), especially in the apical region $(P<0.05)$. Super Bond C\&B (SB) was stronger than the other two cements, sometimes significantly so. The apical region had the lowest bond strength in all circumstances, although the difference was not always statistically significant. Irrigation with EDTA improved the bond strengths of Panavia $\mathrm{F}(\mathrm{PF})$ and RU significantly in each root region $(P<0.05)$. These results showed that water

\footnotetext{
Corresponding author

" Project supported by the Science and Technology Program of Zhejiang Province (Nos. 2009C33094 and 2013C33126) and the Zhejiang Provincial Natural Science Foundation of China (No. Y2080422)

(C) Zhejiang University and Springer-Verlag Berlin Heidelberg 2014
}

storage decreased the bonding effectiveness of the adhesive resin cements. Irrigation with EDTA after post space preparation was a valuable procedure, especially when used in conjunction with self-etching adhesive or self-adhesive resin cements.

As most bonding failures occur at the dentincement interface rather than at the post-cement interface (Faria e Silva et al., 2007; Radovic et al., 2008), adhesion between the intra-radicular dentin and adhesive resin cements is considered the weak link (Boschian Pest et al., 2002). It is generally accepted that the efficacy of a dentin adhesive depends mostly on the removal of the smear layer and formation of a hybrid layer between the adhesive resin cement and the exposed collagen fibrils within the demineralized dentin (Pashley et al., 1993; Vichi et al., 2002). Adhesive resin cements developed over the past two decades have resulted in improved bond strength, at least when tested shortly after adhesion (Zhang et al., 2008; Bergoli et al., 2012), but the durability of bond strength is equivocal and remains an important issue (Kitasako et al., 2001; Toledano et al., 2007).

Exposure to water is known to degrade dentin bonding (Armstrong et al., 2001). Inadequate infiltration of the adhesive resin cements into wet, hydroxyapatite-depleted collagen may result in incomplete hybridization (Hashimoto et al., 2000). This zone is thought to consist of exposed, unprotected collagen fibrils surrounded by nanometer-sized spaces which permit nanoleakage (Li et al., 2001). The term nanoleakage indicates the uptake of molecules into nanometer-sized spaces present in the hybrid layer in the absence of an interfacial gap. This area may be susceptible to long-term degradation due to the slow hydrolysis of unprotected collagen, reducing the durability of the bond strength. 
To improve the bonding effectiveness of adhesive resin cements to the intra-radicular dentin, chemical irrigation has been recommended to pretreat the intraradicular dentin after post space preparation (Coniglio et al., 2008; Zhang et al., 2008). Irrigation with EDTA, known for its mild demineralization and low abrasion on dentinal substrates, has been reported to remove the smear layer effectively (Coniglio et al., 2008). Our previous study revealed that EDTA could effectively improve the bond strength of a selfetching adhesive resin cement (Panavia F) (Gu et al., 2009) compared to $\mathrm{NaOCl}$ or $\mathrm{NaCl}$. Although many adhesive resin cements bond well to intra-radicular dentin (Boschian Pest et al., 2002; Bitter et al., 2009), the durability of dentin bonding remains a concern.

The purpose of the present study was to evaluate the bonding durability of three different bonding systems: a total-etching adhesive system, Super Bond $\mathrm{C} \& \mathrm{~B}$ (SB); a self-etching adhesive system, Panavia F $(\mathrm{PF})$; and a self-adhesive system, RelyX Unicem (RU). The effect of EDTA irrigation on bonding durability in different root regions over six months of water storage was also evaluated. The null hypothesis was that the bond strength would not be affected by water storage, type of adhesive resin cement, EDTA irrigation or root region.

In our study, four-way analysis of variance (ANOVA) revealed that water storage, cement type, post space irrigation and root region significantly affected the push-out bond strength $(P<0.05$; Fig. 1$)$. Water storage reduced the bond strength of RU in the apical region significantly $(P<0.05)$.

Bonding effectiveness was affected by the type of cement used. In the control group, SB showed the highest push-out bond strength. After one week, the bond strength of SB was significantly higher than that of RU in all three regions; at six months, it was significantly higher in the middle and apical regions $(P<0.05)$. PF also showed higher bond strength than RU, but the difference was not significant. In the EDTA group, only RU showed significantly lower bond strength than SB in the apical region at both observation points.

In comparison to the control group, EDTA irrigation produced significantly increased bond strength in the PF and RU subgroups in each root region $(P<0.05)$, and in the apical region in the SB subgroup at one week.

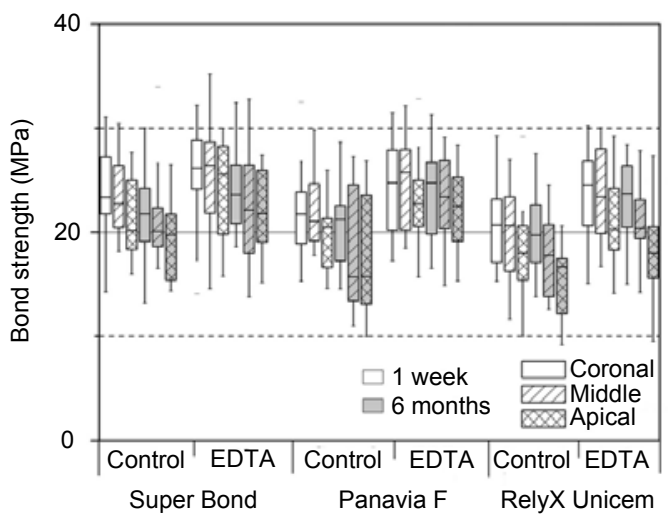

Fig. 1 Push-out bond strength of three adhesive resin cements in three root regions (coronal, middle, and apical) with EDTA irrigation and water storage, at one week and six months

Water storage reduced the bond strength, but only RelyX Unicem (RU) showed a significant difference in the apical region $(P<0.05)$. Super Bond $\mathrm{C} \& \mathrm{~B}(\mathrm{SB})$ showed significantly higher bond strength than RU $(P<0.05)$. The bond strengths of Panavia F (PF) and RU were improved significantly in each root region after EDTA irrigation $(P<0.05)$. In the control group, the lowest bond strength was recorded in the apical region for all three cements $(P<0.05)$. In the EDTA group, only RU had significantly lower bond strength in the apical region $(P<0.05)$

Push-out bond strength was affected by the root region. In the control group, the lowest bond strength was recorded in the apical region for all three cements $(P<0.05)$. In the EDTA group, however, only RU had significantly lower bond strength in the apical region than in the middle and cervical regions at one week, and lower bond strength in the apical region than in the cervical region at six months $(P<0.05)$.

Typical micrographs of fracture interfaces are shown in Fig. 2. With the use of SB, a thick hybrid layer and prominent resin tags were observed. Fractures tended to occur at the top of the hybrid layer. In the PF group, appropriate hybrid layer and resin tags were observed. However, in the RU group, the hybrid layer was weak, and the fracture occurred at the bottom of the layer. There was less infiltration of resin tags.

Push-out bond strength decreased after six months of water storage, although differences were not significant, except for RU in the apical region. A decrease in bond strength after extended water storage is believed to result from hydrolysis of the resin and degradation of exposed collagen fibrils in the resin-dentin interdiffusion zone (RDIZ). Hydrolysis is a chemical process which occurs only in the presence of water, and degradation of the collagen fibrils 

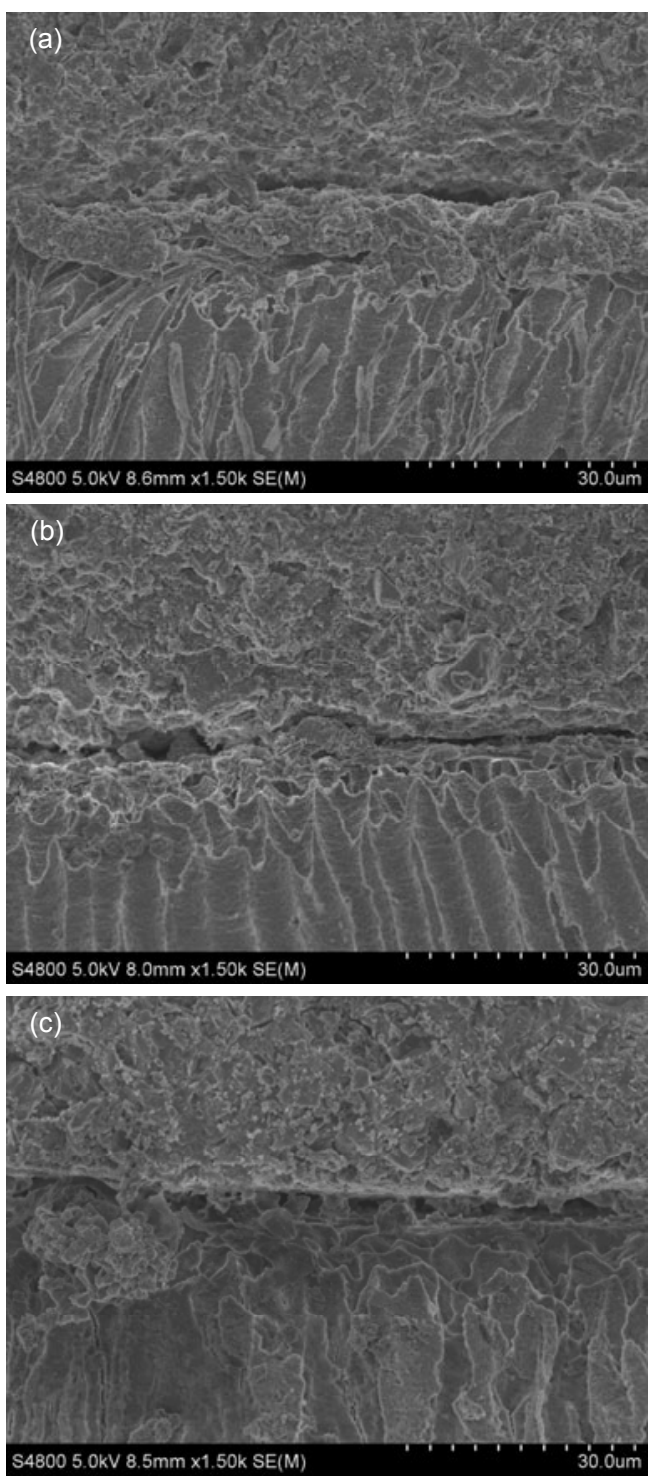

Fig. 2 Scanning electron micrograph of fracture interfaces from three different adhesive resin cements (a) SB group: the fracture occurred at the top of the hybrid layer, and prominent resin tags were observed; (b) PF group: appropriate hybrid layer and resin tags were observed; (c) RU group: fracture occurred at the bottom of the hybrid layer, with less infiltration of resin tags

in the RDIZ occurs only when fibrils are exposed to water (Hashimoto et al., 2000; 2003). Incomplete infiltration and hydrolysis of the resin could leave collagen fibrils unprotected and vulnerable, leading to degradation of the collagen fibrils (Hashimoto et al., 2000). Therefore, complete permeation and envelopment of the collagen fibrils is important to protect the adhesion from degradation. The degree of en- capsulation partially depends on the type of cement used, as the components of different cements produce variable demineralizing effects. As RU has a low effect on demineralization, a thick smear layer remaining on the intra-radicular dentin may prevent the demineralization and infiltration of the cement. This may explain why only RU showed a significant difference after storage.

Generally, bond strength was affected by the type of cement used. SB showed the highest bond strength. This result may be explained by the use of its activator, which dissolves the thick smear layer and debris on the intra-radicular dentin surface. This process leads to adequate intra-radicular dentin demineralization, so that the resin cement can infiltrate the dentinal tubules and encapsulate the exposed collagen network, forming a thick hybrid layer with a stout micromechanical interlocking of cement and dentin (Yang et al., 2006). In the present study, PF showed higher bond strength than RU, although the two did not differ significantly. Moreover, bond strength did not decrease significantly after six months of water storage. PF is a "mild" self-etching adhesive system in which the smear layer is partially dissolved by the primer, which contains an acidic monomer (Rathke et al., 2007). Therefore, the hybrid layer of PF is a combined structure consisting of resin, collagen fibrils and the remains of the smear layer. The functional monomer 10-methacryloyloxydecyl dihydrogen phosphate (10-MDP) of PF interacts chemically with the hydroxyapatite left around the collagen fibrils within the hybrid layer (Fukegawa et al., 2006). This interaction may help the interfacial polymerization of the cement, and aid the long-term stability of the bond (Inoue et al., 2005).

EDTA irrigation significantly improved both the short- and long-term bond strengths in each root region for PF and RU. This result agrees with the findings of our previous study (Gu et al., 2009). EDTA has been shown to dissolve and eliminate the smear layer and debris (Pérez-Heredia et al., 2006; Gu et al., 2009), leaving the intra-radicular dentin surface with demineralized collaged matrices (Calt and Serper, 2002; Garcia-Godoy et al., 2005). Habelitz et al. (2002) found that EDTA could selectively eliminate hydroxyapatite and non-collagenous protein. Unaltered collagen fibrils are more stable and less affected by dehydration. 
In this study, push-out bond strength was affected by the root region, with the lowest values recorded in the apical region. This result is in agreement with previous studies (Perdigão et al., 2004; Faria e Silva et al., 2007). The narrow and deep characteristics of the post space in this region may explain this result. Weakness could also be caused by the difficulty in accessing the apical region or restriction of the flow of adhesive resin cement (de Durâo Mauricio et al., 2007). However, some studies found no significant difference among root regions (Zhang et al., 2008; Babb et al., 2009). In our previous study, although we observed lower bond strength for PF in the apical region, the difference was not significant $(\mathrm{Gu}$ et al., 2009). These conflicting findings may indicate that adhesive procedures performed in the apical region are challenging and sensitive to the technology used.

In conclusion, the null hypothesis that the bond strength would not be affected by water storage, type of adhesive resin cement, use of EDTA irrigation, or root region is rejected. Water storage may play an important and deleterious role in degradation of bonding effectiveness. Irrigation with EDTA after post space preparation can effectively improve bond strength. Such a procedure is valuable during the post-and-core technique, especially when used with self-etching adhesive or self-adhesive resin cements.

\section{Materials and methods}

\section{Tooth preparation}

One hundred and twenty sound, human, singlerooted teeth were used in the study. Teeth were extracted for periodontal reasons. Patients ranged in age from 35 to 60 years.

The extracted teeth were stored in $0.1 \%$ thymol solution and were used within three months of extraction. Standardized endodontic treatment for all teeth was performed chemo-mechanically, establishing a working length of $0.5-1.00 \mathrm{~mm}$ short of the apex. A standardized step-back technique was used, employing k-type files (Dentsply, York, PA, USA) to create root canals of ISO size 60 , rinsing intermittently with $0.025 \mathrm{~g} / \mathrm{ml}$ sodium hypochloride. After drying with paper points (Densply), all roots canals were obturated with a resin sealer (AH plus, DeTrey,
Constance, Germany) and gutta-percha points (Dentsply) using lateral condensation.

After storage for two weeks in a freshly mixed thymol solution at $37^{\circ} \mathrm{C}$, the anatomic crown of each tooth was cut at $2 \mathrm{~mm}$ incisally to the cementoenamel junction. Post space was prepared with low-speed post drills (Snowpost, Carbotech, Ganges, France) under running water, to a length of $10 \mathrm{~mm}$ and a diameter of 1.5 or $1.6 \mathrm{~mm}$ depending on the tooth diameter, leaving about 4-5 $\mathrm{mm}$ of gutta-percha to preserve the apical seal. Each root was attached to a fiber post of compatible diameter with a drop of sticky wax, and the post was mounted to a paralleling fixture, to ensure that each root was sectioned perpendicularly. A vitric mold was placed centrally below the fixture, and then embedded in self-curing acrylic resin. At the same time, the post space was positioned centrally in the mold. After the resin had cured fully, the fiber post was removed.

The samples were then randomly assigned to two groups of 60 teeth each, according to post space pre-treatment: (1) control group: the root canal dentin was irrigated with deionized water for $60 \mathrm{~s}$; (2) treatment group: irrigation with $14 \%$ EDTA for $60 \mathrm{~s}$. All samples were dried lightly with paper points. Each group was then divided into three subgroups of 20 teeth each, according to which adhesive resin cement the group received: (1) a total-etching adhesive system, SB (Sun Medical Co., Ltd., Moriyama City, Japan); (2) a self-etching adhesive system, PF (Batch No. 71127, Kuraray Chemical Co., Ltd., Osaka, Japan); or (3) a self-adhesive system, RU (3M ESPE AG, Neuss, Germany). Each of the adhesive resin cements was applied following the manufacturer's instructions. After the cements were fully cured, all samples were stored in deionized water at $37{ }^{\circ} \mathrm{C}$ until observation. Ten samples of each subgroup were observed after one week and the other ten after six months.

\section{Push-out test}

Specimens were serially sectioned transversally from $1 \mathrm{~mm}$ below the coronal surface to the apex, using a low-speed diamond saw (Isomet 1000, Buehler, Lake Bluff, IL) under running water as a coolant. From each root, six $1 \mathrm{~mm}$-thick serial slices were obtained: two each of the coronal, middle and apical root sections. Bond strength was evaluated by a 
push-out test. All slices were tested with the apical surface facing up, using a universal testing machine (Z010/TN2S, Zwick GmbH and Co., Ulm, Germany), at a cross-head speed of $0.5 \mathrm{~mm} / \mathrm{min}$. The load was applied in the apical-coronal direction until bond failure, and then the maximum failure load was recorded. The push-out bond strength $(\sigma)$, expressed as $\mathrm{MPa}$, was calculated according to the formula of $\sigma=F / \pi(r+R) h$, where $\pi$ is the constant $3.14, r$ and $R$ represent the radius of the luting cement surfaces on each cut surface of the slice, $h$ represents the height of the slice, and $F$ represents the maximum failure load (Gu et al., 2009).

\section{Observation of fracture interface}

After the push-out test, the samples were decalcified with $0.35 \mathrm{~g} / \mathrm{ml}$ phosphoric acid for $30 \mathrm{~s}$ and deproteinized with $0.02 \mathrm{~g} / \mathrm{ml}$ sodium hypochlorite solution for $120 \mathrm{~s}$ to observe the fracture interface under the scanning electron microscope (SEM, S-4800; Hitachi, Tokyo, Japan).

\section{Statistical analyses}

The statistical analyses were conducted using SPSS version 16.0 (SPSS Inc., Chicago, IL). The data were verified by the Kolmogorov-Smirnov test for the normal distribution and by Levene's test for the homogeneity of variances. The push-out bond strength in each group was performed using four-way ANOVA. The dependent variable was push-out bond strength and the main effects were water storage, cement type, post space irrigation, and root region. Multiple comparisons between the groups were performed using Tukey's test. $P$-values of $\leq 0.05$ were used as the threshold for significance in all tests.

\section{Compliance with ethics guidelines}

Cai-yun MAO, Jia-jia ZHAO, Wei WANG, and Xin-hua GU declare that they have no conflict of interest.

All procedures followed were in accordance with the ethical standards of the committees responsible for human experimentation (institutional and national) and with the Helsinki Declaration of 1975, as revised in 2008 (5). Informed consent was obtained from all patients for being included in the study.

\section{References}

Armstrong, S.R., Keller, J.C., Boyer, D.B., 2001. The influence of water storage and $\mathrm{C}$-factor on the dentin-resin composite microtensile bond strength and debond pathway utilizing a filled and unfilled adhesive resin. Dent. Mater., 17(3): 268-276. [doi:10.1016/S0109-5641(00)00081-6]

Babb, B.R., Loushine, R.J., Bryan, T.E., et al., 2009. Bonding of self-adhesive (self-etching) root canal sealers to radicular dentin. J. Endod., 35(4):578-582. [doi:10.1016/ j.joen.2009.01.005]

Bergoli, C.D., Amaral, M., Boaro, L.C., et al., 2012. Fiber post cementation strategies: effect of mechanical cycling on push-out bond strength and cement polymerization stress. J. Adhes. Dent., 14(5):471-478. [doi:10.3290/j.jad.a28389]

Bitter, K., Paris, S., Pfuertner, C., et al., 2009. Morphological and bond strength evaluation of different resin cements to root dentin. Eur. J. Oral Sci., 117(3):326-333. [doi:10. 1111/j.1600-0722.2009.00623.x]

Boschian Pest, L., Cavalli, G., Bertani, P., et al., 2002. Adhesive post-endodontic restorations with fiber posts: push-out tests and SEM observations. Dent. Mater., 18(8): 596-602. [doi:10.1016/S0109-5641(02)00003-9]

Calt, S., Serper, A., 2002. Time-dependent effects of EDTA on dentin structures. J. Endod., 28(1):17-19. [doi:10.1097/ 00004770-200201000-00004]

Coniglio, I., Magni, E., Goracci, C., et al., 2008. Post space cleaning using a new nickel titanium endodontic drill combined with different cleaning regimens. J. Endod., 34(1):83-86. [doi:10.1016/j.joen.2007.10.019]

de Durâo Mauricio, P.J., González-López, S., Aguilar-Mendoza, J.A., et al., 2007. Comparison of regional bond strength in root thirds among fiber-reinforced posts luted with different cements. J. Biomed. Mater. Res. B Appl. Biomater., 83B(2): 364-372. [doi:10.1002/jbm.b.30805]

Faria e Silva, A.L., Casselli, D.S., Ambrosano, G.M., et al., 2007. Effect of the adhesive application mode and fiber post translucency on the push-out bond strength to dentin. J. Endod., 33(9):1078-1081. [doi:10.1016/j.joen.2007.03. 018]

Fukegawa, D., Hayakawa, S., Yoshida, Y., et al., 2006. Chemical interaction of phosphoric acid ester with hydroxyapatite. J. Dent. Res., 85(10):941-944. [doi:10. 1177/154405910608501014]

Garcia-Godoy, F., Loushine, R.J., Itthagarun, A., et al., 2005. Application of biologically-oriented dentin bonding principles to the use of endodontic irrigants. Am. J. Dent., 18(4):281-290.

Gu, X.H., Mao, C.Y., Liang, C., et al., 2009. Does endodontic post space irrigation affect smear layer removal and bonding effectiveness? Eur. J. Oral Sci., 117(5):597-603. [doi:10.1111/j.1600-0722.2009.00661.x]

Habelitz, S., Balooch, M., Marshall, S.J., et al., 2002. In situ atomic force microscopy of partially demineralized human dentin collagen fibrils. J. Struct. Biol., 138(3): 227-236. [doi:10.1016/S1047-8477(02)00029-1]

Hashimoto, M., Ohno, H., Kaga, M., et al., 2000. In vivo degradation of resin-dentin bonds in humans over 1 to 3 years. J. Dent. Res., 79(6):1385-1391. [doi:10.1177/ 00220345000790060601]

Hashimoto, M., Ohno, H., Sano, H., et al., 2003. In vitro 
degradation of resin-dentin bonds analyzed by microtensile bond test, scanning and transmission electron microscopy. Biomaterials, 24(21):3795-3803. [doi:10.1016/ S0142-9612(03)00262-X]

Inoue, S., Koshiro, K., Yoshida, Y., et al., 2005. Hydrolytic stability of self-etch adhesives bonded to dentin. J. Dent. Res., 84(12):1160-1164. [doi:10.1177/15440591050840 1213]

Kitasako, Y., Burrow, M.F., Katahira, N., et al., 2001. Shear bond strengths of three resin cements to dentine over 3 years in vitro. J. Dent., 29(2):139-144. [doi:10.1016/ S0300-5712(00)00058-0]

Li, H.P., Burrow, M.F., Tyas, M.J., 2001. The effect of long-term storage on nanoleakage. Oper. Dent., 26(6): 609-616.

Pashley, D.H., Ciucchi, B., Sano, H., et al., 1993. Permeability of dentin to adhesive agents. Quintessence Int., 24(9): 618-631.

Perdigão, J., Geraldeli, S., Lee, I.K., 2004. Push-out bond strengths of tooth-colored posts bonded with different adhesive systems. Am. J. Dent., 17(6):422-426.

Pérez-Heredia, M., Ferrer-Luque, C.M., González-Rodríguez, M.P., 2006. The effectiveness of different acid irrigating solutions in root canal cleaning after hand and rotary instrumentation. J. Endod., 32(10):993-997. [doi:10. 1016/j.joen.2006.05.016]
Radovic, I., Mazzitelli, C., Chieffi, N., et al., 2008. Evaluation of the adhesion of fiber posts cemented using different adhesive approaches. Eur. J. Oral Sci., 116(6):557-563. [doi:10.1111/j.1600-0722.2008.00577.x]

Rathke, A., Alt, A., Gambin, N., et al., 2007. Dentin diffusion of HEMA released from etch-and-rinse and self-etch bonding systems. Eur. J. Oral Sci., 115(6):510-516. [doi:10.1111/j.1600-0722.2007.00484.x]

Toledano, M., Osorio, R., Osorio, E., et al., 2007. Durability of resin-dentin bonds: effects of direct/indirect exposure and storage media. Dent. Mater., 23(7):885-892. [doi:10. 1016/j.dental.2006.06.030]

Vichi, A., Grandini, S., Ferrari, M., 2002. Comparison between two clinical procedures for bonding fiber posts into a root canal: a microscopic investigation. J. Endod., 28(5):355-360. [doi:10.1097/00004770-200205000-00002]

Yang, B., Ludwig, K., Adelung, R., et al., 2006. Micro-tensile bond strength of three luting resins to human regional dentin. Dent. Mater., 22(1):45-56. [doi:10.1016/j.dental. 2005.02.009]

Zhang, L., Huang, L., Xiong, Y., et al., 2008. Effect of post-space treatment on retention of fiber posts in different root regions using two self-etching systems. Eur. J. Oral Sci., 116(3):280-286. [doi:10.1111/j.1600-0722. 2008.00536.x]

\section{中文概要:}

本文题目: 乙二胺四乙酸处理和水储存对不同树脂粘结剂与根管牙本质间粘结耐久性的影响

Effects of EDTA irrigation and water storage on the bonding durability of different adhesive resin cements to intra-radicular dentin

本文概要：树脂粘结材料已广泛应用于临床，但其粘结耐久性仍不尽人意，导致牙本质粘结修复体远期 存在继发龊、脱落率高等问题，成为目前亟需解决的关键问题。本文通过探讨不同树脂粘结 剂（Supper Bond C\&B、Panavia F 和 RelyX Unicem）与根管牙本质间粘结耐久性的差异，以 及乙二胺四乙酸（EDTA）处理对不同树脂粘结剂粘结耐久性的影响, 发现 EDTA 处理可以 提高树脂粘结剂与根管牙本质间的粘结强度和粘结耐久性, 从而可以提高修复体的远期粘结 效果。另外, 水储存可造成树脂粘结剂与根管牙本质间粘结强度下降。

关键词组：水储存；乙二胺四乙酸；树脂粘结剂；粘结耐久性 